\title{
Atraumatic Removal of Displaced Implant in the Fatty Bony Marrow
}

\author{
Buyanbileg Sodnom-Ish ${ }^{1+}$, Mi Young Eo ${ }^{1+}$, Truc Thi Hoang Nguyen', Yun Ju Cho', \\ Myung Joo Kim², Soung Min Kim ${ }^{1 *}$ \\ 'Department of Oral and Maxillofacial Surgery, Dental Research Institute, School of Dentistry, Seoul National \\ University, Seoul, Korea \\ ${ }^{2}$ Department of Prosthodontics, Dental Research Institute, School of Dentistry, Seoul National University, Seoul, \\ Korea \\ *Corresponding author: Soung Min Kim, smin5@snu.ac.kr, smin_kim@hanmail.net \\ ${ }^{\dagger}$ Equal co-first authors.
}

\section{OPEN ACCESS}

pISSN 1229-5418

eISSN 2671-6623

Implantology 2019; 23(3): 176-183

https://doi.org/10.32542/implantology.2019015

Received: August 23, 2019

Revised: September 16, 2019

Accepted: September 19, 2019

ORCID

Buyanbileg Sodnom-Ish

https://orcid.org/0000-0003-1137-3673

Mi Young Eo

https://orcid.org/0000-0001-7055-9924

Truc Thi Hoang Nguyen

https://orcid.org/0000-0002-8667-6698

Yun Ju Cho

https://orcid.org/0000-0002-1818-5280

Myung Joo Kim

https://orcid.org/0000-0003-2020-5284

Soung Min Kim

https://orcid.org/0000-0002-6916-0489

Copyright $($ 2019. The Korean Academy of Oral \& Maxillofacial Implantology

This is an Open Access article distributed under the terms of the Creative Commons Attribution Non-Commercial License (http://creativecommons. org/licenses/by-nc/4.0/) which permits unrestricted non-commercial use, distribution, and reproduction in any medium, provided the original work is properly cited.

\section{Abstract}

A 53-year-old Korean female was referred to the Department of Oral and Maxillofacial Surgery at the Seoul National University Dental Hospital. The patient's chief complaint and concern involved the occasional feeling of discomfort and pain in the region of the lower right second molar. Panoramic radiograph revealed a \#47 implant fixture that was dislocated horizontally deep in the fatty degenerated area of the posterior mandible. A rare case of implant displacement into the fatty degenerated area of the posterior mandible caused inferior alveolar nerve (IAN) compression. Fatty degeneration is a condition that occurs in mostly elderly female patients due to aging and is inevitable. We recommend accurate preoperative evaluation using radiographic imaging techniques such as computer tomography and histopathological analysis for differential diagnosis. Endoscopic intraoperative examinations can reduce the invasiveness of the surgery and provide accuracy in examining the intactness of the IAN. This case report suggests the efficacy of the selected method of treatment and, the removal of the displaced implant and decompression of the IAN using endoscopic visualization followed by 8 months of healing and two-stage implant installation with guided bone regeneration in cases of related implant complications.

Keywords: Dental implant, fatty degeneration, bone marrow, endoscopic visualization

\section{I . Introduction}

Dental implantation is a treatment modality that replaces missing teeth in partial or fully edentulous patients and has high success and survival rates ${ }^{1,2}$. Despite its commercial use rare risks and complications associated with surgical and restorative phases of the treatment have been reported ${ }^{1,3,4}$. One of the complications that may occur is the displacement of implants during the intraoperative phase or within a short period of time. On the other hand, migration of implants can occur due to long-term relative motion. In either case, the bone quality and quantity is compromised ${ }^{5}$. Dental implant displacement can cause serious 
injury to the inferior alveolar nerve (IAN) that can additionally undergo secondary injury during the surgical retrieval of these displaced implants.

Marrow fat increases with age, particularly in the third decade of life. This increase in bone marrow fat (BMF) differs between genders, being steeper in women, whereas in men it increases gradually with age. Therefore, BMF is higher in women at an older age ${ }^{5}$. Several studies have revealed a positive correlation between implant failure and low bone density ${ }^{1,5,6}$. Most cases of displaced of dental implants were observed in older women in the medullary space of the posterior mandible with focal osteoporotic bone marrow defects ${ }^{1,3,6}$. Our present case reports a case of dental implant displacement into the fatty degenerated bone marrow of the posterior mandible in a female patient of menopausal age.

\section{II . Case report}

A 53-year-old Korean female was referred to the Department of Oral and Maxillofacial Surgery at the Seoul National University Dental Hospital from a local dental clinic. The patient's chief complaint and concerns were occasional discomfort and pain in the lower right second molar implant site caused by implant fixture instability. Before patient came, local doctor made an urgent phone call, and explained that he did not find the lost fixture in the hollow and blood filled bony marrow cavity, in spite of his trial to find it after implant fixture installation with mounter-free grasping. The patient requested the elimination of her current problems and to restore her missing tooth for function, esthetics, and confidence. Anamnesis was negative for any serious medical conditions such as osteoporosis.

Upon clinical examination, tooth \#47 was missing and the surrounding tissue was in a healthy state upon inspection. A panoramic radiograph was taken for a general overview of the underlying bony structures and pathological findings. According to the radiographic evaluations, the \#47 implant fixture was horizontally displaced deep in the fatty degenerated region of the posterior mandible. The patient's panoramic radiograph displayed a generalized reduced radiopacity and weakened bone marrow with D4 quality bone surrounding the foreign body (Fig. 1A). In this case, IAN compression could be expected considering the chief complaint and radiographic finding caused by the proximity of the displaced implant fixture to the inferior alveolar nerve canal (IANC). However, fortunately, the patient did not complain of moderate or severe paresthesia of involved skin or right mandibular area.

Based on clinical and radiographic examinations, the following treatment plan was proposed as: (1) Removal of the displaced dental implant fixture and decompression of the IAN immediately, followed by a healing period; (2) After 6-8 months of healing, implant installation along with guided bone 
regeneration (GBR); and (3) Final delivery of the implant prosthesis. With careful consultation with the patient, considering her needs and expectations, the patient agreed to the proposed treatment plan.

IAN block and infiltration anesthesia were administered on that day urgently. A full-thickness flap was raised with molt and periosteal elevators easily due to its sutured state from a local clinic. Crushed marrow cavity was found with a blood clot, and the implant fixture was carefully removed without additional damage by suction tip under the endoscopic views (Fig. 1B) since it was in a mobilized state in type D4 bone quality. We used 0 and 70 degree endoscope ${ }^{\circledR}$ (Karl Storz Co., Tuttlingen, Germany) with a $2.7 \mathrm{~mm}$ diameter. Further endoscopic examination was performed to assess the damage to the IAN, and the IAN was intact in spite of in a compressed state as a result of the mechanical pressure of the fixture. The removed implant was considered as tapered fixture with $7.0 \mathrm{~mm}$ length and $6.0 \mathrm{~mm}$ platform diameter. Fixture seemed to have intact surface without any attached mounter or cover screw. The mucoperiosteal flap was placed back in its original position and sutured with drainage (Fig. 1C). The patient received standard postoperative care and regular follow-up two, three, and eight months postoperative for the assessment of healing progress. Following the removal of the implant fixture and decompression procedure of the IAN, the damage to the buccal wall could not be avoided (Fig. 1D).

The patient was scheduled to undergo the first stage of implant installation along with autogenous and allogeneic bone grafts, due to alveolar ridge dehiscence on the buccal side of the implant site. Implant placement and GBR was performed under local anesthesia. A crestal incision created using no. 12 and 15 blades and a full-thickness flap was elevated in the \#47 area (Fig. 2A). To fill the hard tissue defect and improve the alveolar ridge contour on the buccal surface, an autologous bone chip was harvested from the right maxillary tuberosity (Fig. 2B). The implant bed was prepared with a standard osteotomy and measurements were performed to determine the depth of implant placement. The right maxillary tuberosity was chosen as an intraoral bone harvesting site for the buccal alveolar ridge defect. A full
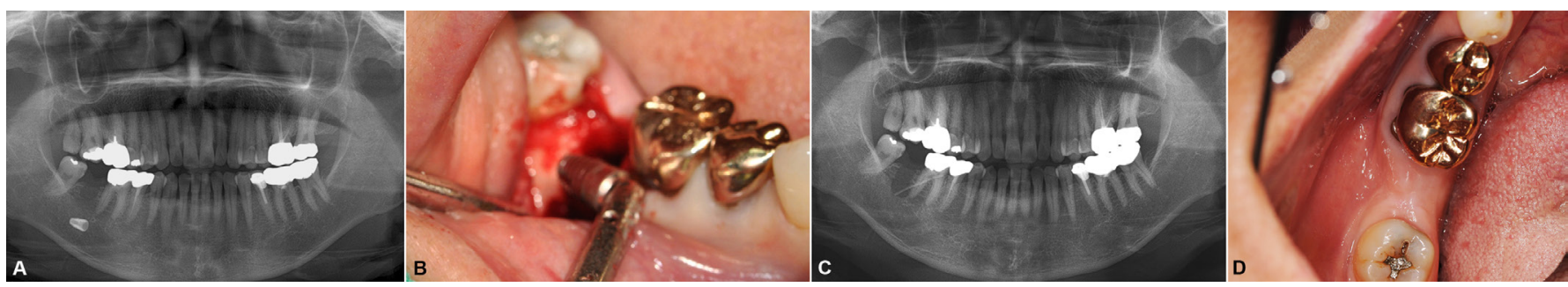

Fig. 1. A. Pre-operative radiograph revealed the \#47 implant located horizontally in the deep fatty degenerated fatty bone marrow. An irregular radiolucent area can be observed at the initial position of the implant fixture, B. The implant was removed without additional damage by the suction tip under endoscopic examination, C. Immediate postoperative radiograph exhibit careful removal of the implant with drainage placement, D. Eight-month postoperative clinical view exhibiting buccal dehiscence of the alveolar bone.

Buyanbileg Sodnom-Ish et al. : Atraumatic Removal of Displaced Implant in the Fatty Bony Marrow. Implantology 2019 
buccal thickness flap was elevated to expose the right posterior maxillary tuberosity area. An autogenous bone chip was harvested with chisels and the full thickness flap was sutured into place. For better projection of the buccal bone and expected bone resorption, the initial layer of the harvested autogenous bone chip was placed in the deficient area along the buccal contour (Fig. 2C). A $10.0 \mathrm{~mm}$ length SLActive ${ }^{\circledR}$ tissue level (TL) implant (Straumann ${ }^{\circledR}$ Holding AG, Basel, Switzerland) was placed in the \#47 area (Fig. 2D). After implant installation, an allogeneic Oragraft ${ }^{\circledR}$ (LifeNet Health Co, VA, USA) particulate bone was grafted in combination with the autogenous bone chip as a second layer (Fig. 2E). The flap was closed with a tension-free suture using 4-0 Vicry ${ }^{\circledR}$ (Polyglactin 910, Johnson \& Johnson Co., USA). The patient received standard postoperative care as the first procedure and the stitches were removed 7 days later. The panoramic radiograph evaluation of the postoperative state showed a well osseointegrated immobile implant fixture with integrated grafting materials (Fig. 2F). The patient reported uneventful healing.

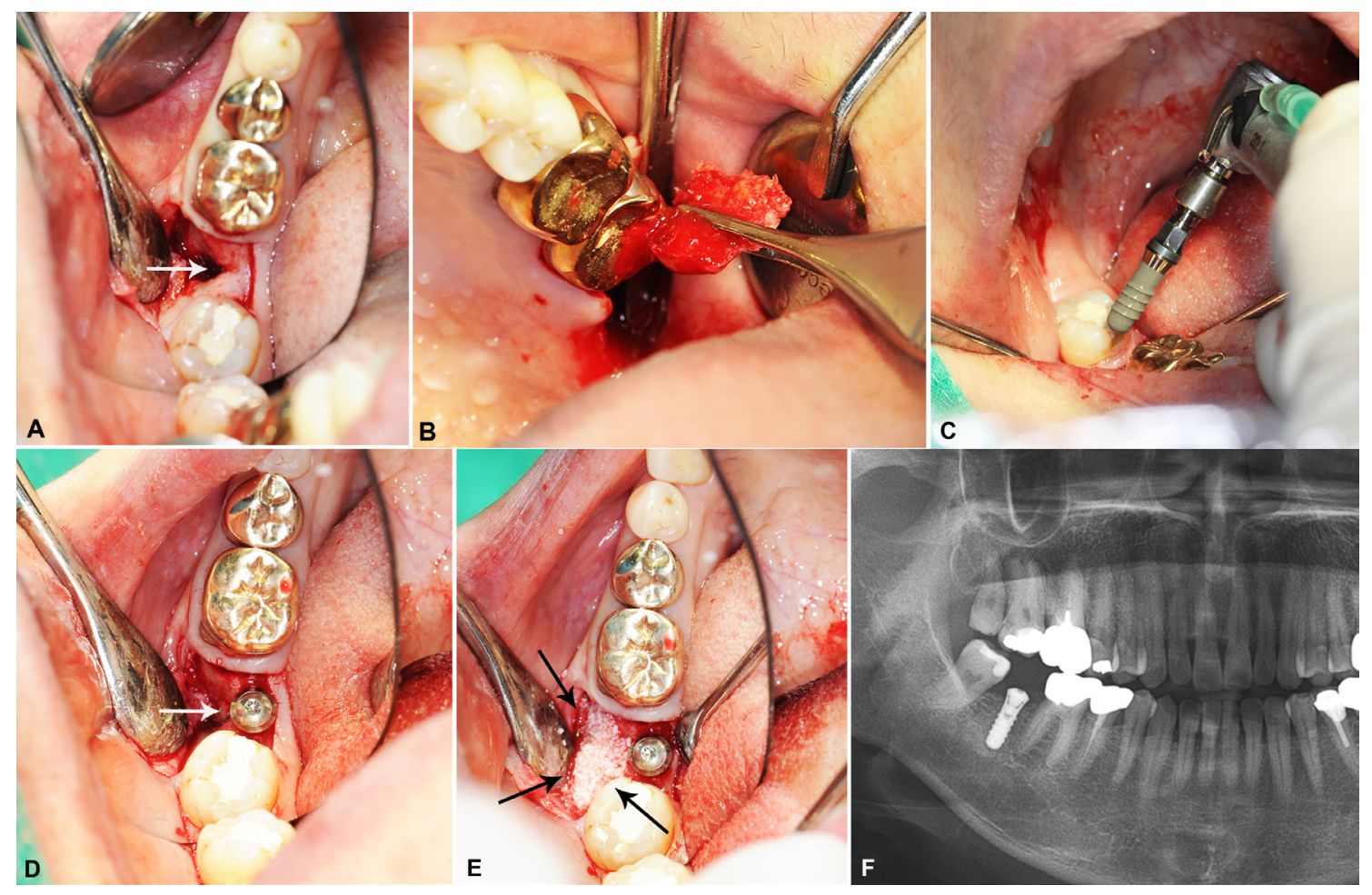

Fig. 2. A. An intraoral view of the alveolar ridge defect after full-thickness flap elevation (marked by arrow), B. Autogenic

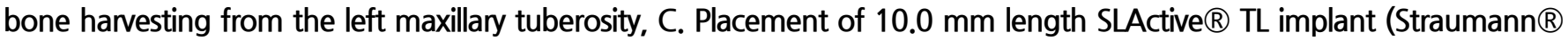
Holding AG, Basel, Switzerland) in the \#47 area, D. Position of the autogenous maxillary tuberosity chip and the buccal wall defect with implant placement (marked by white arrow), E. Remained defect space was filled with allogeneic Oragraft $®$ (LifeNet Health Co, VA, USA) particulate bone in combination with the autogenous bone chip (marked by black arrows), F. Immediate radiograph after GBR and implant installation. A well osseointegrated immobile implant fixture with integrated grafting materials can be observed. 

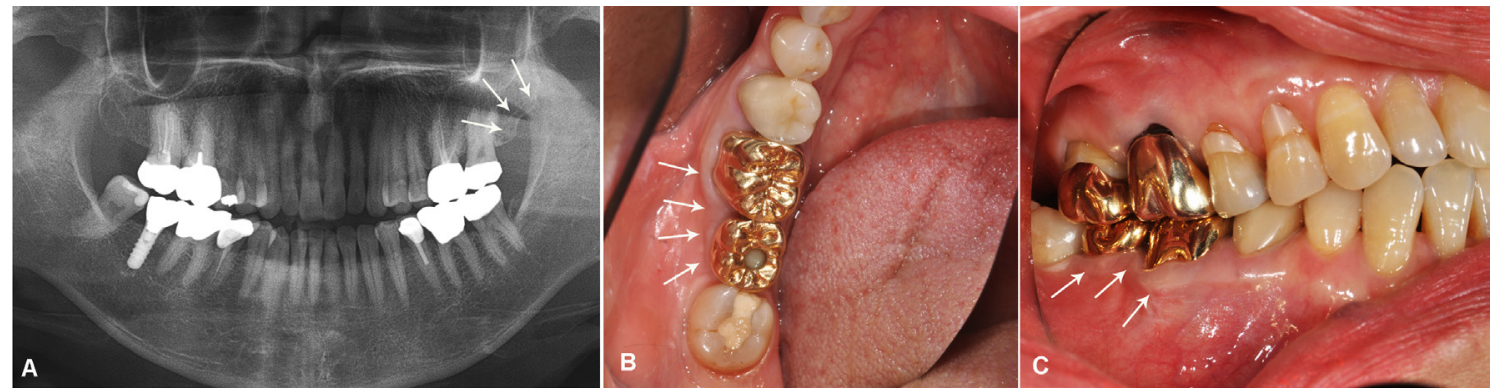

Fig. 3. A. Panoramic view showing the maxillary tuberosity harvesting site (marked by arrows), B. Intraoral view showing the clinical outcome (marked by arrows), C. Keratinized gingival line can be seen six years after implant placement at the augmented site (marked by arrows). The implant and prostheses showed good function and esthetic status.

Buyanbileg Sodnom-Ish et al. : Atraumatic Removal of Displaced Implant in the Fatty Bony Marrow. Implantology 2019

After 5 months of healing, the second stage of minimally invasive reentry was performed under local anesthesia with a small incision and healing abutment was connected to the implant. Following reentry after 3 months, the final prosthetic restoration on the \#47 implant fixture was delivered. A panoramic radiograph was taken at completion 1 year later and the patient was reexamined for follow-up and reported no significant changes. During the follow-up period, on clinical and radiographic evaluations, the reimplantation procedure along with GBR augmentation exhibited stable outcomes. The patient had regular long-term follow-up visits for six years up until 2019. The end result after six years was satisfactory from both the perspective of the clinician and patient (Fig. 3). The patient did not report any pain or discomfort.

\section{Discussion}

Our present patient had type IV (mandible) bone quality during the implant placement procedure. Due to fatty degeneration of the posterior mandible, the implant was displaced horizontally when primary stability was lost. Complications associated with implant placement are mostly related to the loss of primary stability and its subsequent implant failure. Possible factors contributing to the loss of primary stability are anatomical variances such as decreased cortical bone thickness, low trabecular bone density, poor bone quality, osteoporosis, and osteopenia. Another group of factors is related to the surgical technique operated by an inexperienced surgeon, inadequate planning, overheating of the implant bed, mishandling, and incorrect manipulation ${ }^{1,7}$. In our case, inadequate bone quality due to fatty degeneration was a possible factor in implant displacement. The main cause of implant displacement in the posterior mandible was believed to be osteoporosis, however, articles suggesting osteoporosis as a probable cause 
of displacement lacked evidence ${ }^{7}$. Similarly, our patient did not exhibit any sign of osteoporosis.

Another main cause was focal osteoporotic bone marrow defects, or the presence of uncommon hematopoietic tissue in the posterior molar region of the mandible in middle-aged women. This condition is usually asymptomatic and mostly appears in edentulous posterior mandibles where tooth extraction has previously been performed. The displacement of dental implants has been reported in a few cases with osteoporotic bone marrow defects ${ }^{1,7}$. Most defects occur at former extraction sites. Diagnosis and treatment plans were based on clinical, radiographic, and microscopic findings in these cases. Differential diagnosis is between other intraosseous pathologic conditions such as osteomyelitis, traumatic bone cyst, and fibrous dysplasia. Upon radiographic examination, an isolated or multifocal radiolucency with a variety of sizes ranging from several millimeters to centimeters with poorly defined borders and shape were observed. Garcia et al.reported that upon microscopic examination, hematopoietic marrow composed of monocytic, erythroid, granulocytic, and lymphocytic series with megakaryocytes associated with fatty marrow was detected. The diagnosis can be made based on the distinct features of the condition such as age, site of the defect, and clinical and radiological findings with microscopic evaluation ${ }^{6}$.

Lee et al. reported hypoesthesia to be a possible complication of displacement of dental implants in focal osteoporotic bone marrow defects1. In our case, the IAN was compressed by the displaced dental implant. Endoscopy was performed to visualize and confirm the position and intactness of the IAN at the difficult-to-access surgical site8. Endoscopic visualization of critical intraosseous structures such as IAN improves the quality and reduces the invasiveness of the dentoalveolar surgery.

Age-related bone loss involves an extremely complex mechanism and multiple factors are known to play a critical role in this process. Extensive evidence reveals the correlation between aging and increase of $\mathrm{BMF}^{9}$. Humans and animals share a common feature of aging, the infiltration of adipocytes into bone marrow $^{10}$. The increased amount of BMF is the result of an imbalance between bone formation by osteoblast cells and bone resorption by osteoclast cells. The balance is regulated by the paracrine secretion of fatty acids and adipocytes. In middle-aged women with osteoporosis, low levels of estrogen are associated with the increased differentiation and function of osteoclasts which can facilitate bone resorption. On the other hand, in relation to the decreased number of osteoblasts, adipocytes begin to occupy the space of otherwise normal bone marrow. Osteoblasts and adipocytes are both derived from mesenchymal stem cells (MSC), whereas osteoclasts belong to the macrophage family. MSCs only differentiate into osteoblasts under optimal conditions. However, due to aging, these optimal conditions are lost and adipogenesis predominates over osteoblastogenesis ${ }^{9}$. Multiple studies demonstrate that age- 
related bone loss is associated with increased marrow fat. Justesen et al. reported that marrow adiposity increased from $40 \%$ at 30 years to $68 \%$ at 100 years old in relation to the decrease of bone volume ${ }^{10}$.

To avoid unforeseen complications and rare accidents like what can be seen in our case, additional radiographic evaluations, such as preoperative $\mathrm{CTs}$, may be necessary for patients who had their molars extracted early in life, especially in older women. When the location of the IANC cannot be delineated on panoramic radiographs, large medullary components of the bone can be detected on CT. Theisen et al. reported that $\mathrm{CT}$ could provide data regarding cortical plate thickness, location of the inferior nerve canal, and the presence of medullary spaces. This method of preoperative evaluation could enhance implant treatment and decrease any potential complications ${ }^{11}$. To establish a precise diagnosis, a histopathological analysis can be performed in cases of radiolucent areas in the posterior mandible with or without implants. The diagnosis of the present case was based on the preoperative clinical and radiographic evaluations.

Kim et al. suggest using a crestal approach in cases when the implant is not deeply dislocated and lies above the IAN. This procedure can be carried out by enlarging the drill hole, ensuring the safe removal of the implant, as we did in our case ${ }^{7}$. However, the disadvantages include the limited field of vision, inadequate operation site, potential problems with the future fixation, and greater risk of further displacement. To overcome the disadvantages, we performed an endoscopic examination. The surgical intervention involving the removal of the failed implant can be carried out with enhanced endoscopic visualization, decreasing the risks for further displacement and secondary injury to the IAN. Therefore, we recommend using a technique similar to ours in cases with implant displacement with proximity to the IAN.

\section{IV . Conclusion}

The most important points in the case report were: 1) displacement of dental implants in the posterior mandible region was mostly associated with focal osteoporotic bone marrow defects, 2) bone marrow defects cannot be detected on panoramic radiograph, especially in elderly women with a previous history of tooth extraction, 3) displacement of dental implants is most common in elderly women and usually results in complications such as hypoesthesia due to IAN damage, 4) fatty degeneration is inevitable in the elderly, which is not optimal for MSCs differentiation into osteoblasts and adipogenesis becomes more common, and 5) when using the cortical approach to retrieve displaced implants, an endoscopic examination can overcome the disadvantages associated with the cortical approach. 


\section{Abbreviations}
IAN : inferior alveolar nerve
BMF : bone marrow fat
GBR : guided bone regeneration
MSC : mesenchymal stem cell
IANC : inferior alveolar nerve canal

\section{Acknowledgements}

This study was supported by NRF of Korea funded by the Ministry of Education (2017R1D1A1B03036054).

\section{References}

1. Lee SC, Jeong CH, Im HY, et al. Displacement of dental implants into the focal osteoporotic bone marrow defect: A report of three cases. J Korean Assoc Oral Maxillofac Surg. 2013; 39: 94-99.

2. Goiato MC, dos Santos DM, Santiago JF Jr, et al. Longevity of dental implants in type IV bone: A systematic review. Int J Oral Maxillofac Surg. 2014; 43: 1108-1116.

3. Sencimen M, Delilbasi C, Gulses A, et al. Focal osteoporotic hematopoietic bone marrow defect formation around a dental implant: A case report. Int J Oral Maxillofac Implants. 2011; 26: E1-4.

4. Bravo-Calderón D, Oliveira D, Martins dos Santos W. Bilateral osteoporotic bone marrow defects of the mandible: A case report. Head Face Med. 2012; 8: 22.

5. Radi IA, Ibrahim W, Iskandar SMS, et al. Prognosis of dental implants in patients with low bone density: A systematic review and meta-analysis. J Prosthet Dent. 2018; 120: 668-677.

6. Garcia NG, Barros FB, Carvalho MM, et al. Focal osteoporotic bone marrow defect involving dental implant: A case report. Int J Implant Dent. 2015; 1: 1-3.

7. Kim JW, Paeng JY, Choi SY, et al. Displacement of Dental Implants Into the Mandibular Bone Marrow Space: Cause and Treatment. A Case Study and Literature Review. J Oral Implantol. 2017; 43: 151-157.

8. Beltrán V, Fuentes R, Engelke W. Endoscopic visualization of anatomic structures as a support tool in oral surgery and implantology. J Oral Maxillofac Surg. 2012; 70: e1-e6.

9. Duque G. Bone and Fat Connection in Aging Bone. Curr Opin Rheumatol. 2008; 20: 429-434.

10. Kawai M, de Paula FJ, Rosen CJ. New insights into osteoporosis: the bone-fat connection. J Intern Med. 2012; 272: 317-329.

11. Theisen FC, Shultz RE, Elledge DA. Displacement of a root form implant into the mandibular canal. Oral Surg Oral Med Oral Pathol. 1990; 70: 24-28. 